\title{
Peripheral arterial occlusive disease: Global gene expression analyses suggest a major role for immune and inflammatory responses
}

\author{
Shijun Fu'111, Haiguang Zhao ${ }^{2}$, Jiantao Shi1,11, Arhat Abzhanov ${ }^{3,6}$, \\ Keith Crawford ${ }^{3,9}$, Lucila Ohno-Machado ${ }^{3,8}$, Jianqin Zhou ${ }^{10}$, Yanzhi Du ${ }^{1}$, \\ Winston Patrick Kuo*3,6,7,8, Ji Zhang*1, Mier Jiang*2 and Jason Gang Jin*1,4,5
}

Address: ${ }^{1}$ Institute of Health Sciences, Shanghai Institutes for Biological Sciences, Chinese Academy of Sciences and Shanghai Jiao Tong University School of Medicine, Shanghai, PR China, ${ }^{2}$ Shanghai Ninth People's Hospital, Shanghai Jiao Tong University School of Medicine, Shanghai, PR China, ${ }^{3}$ Laboratory for Innovative Translational Technologies, Harvard School of Dental Medicine, Boston, MA, USA, ${ }^{4}$ ShanghaiBio Corporation, 675 US Highway One, North Brunswick, NJ, USA, ${ }^{5}$ Shanghai Biochip Co., Ltd and National Engineering Center for Biochip at Shanghai, Shanghai, PR China, ${ }^{6}$ Department of Organismic and Evolutionary Biology, Harvard University, Cambridge, MA, USA, ${ }^{7}$ Department of Developmental Biology, Harvard School of Dental Medicine, Boston, MA, USA, ${ }^{8}$ Decision Systems Group, Brigham and Women's Hospital, Harvard Medical School, Boston, MA, USA, 'PDepartment of Orthopedics, Brigham and Women's Hospital, Harvard Medical School, Boston, MA, USA, 10School of Pharmacy, Soochow University, Suzhou, JiangSu, PR China and ${ }^{11}$ Graduate School of the Chinese Academy of Sciences, Shanghai, PR China

Email: Shijun Fu - sjfu@sibs.ac.cn; Haiguang Zhao - hai7787@yahoo.com.cn; Jiantao Shi - jtshi@sibs.ac.cn; Arhat Abzhanov - aabzhano@genetics.med.harvard.edu; Keith Crawford - kdcrawford@partners.org; Lucila OhnoMachado - machado@dsg.harvard.edu; Jianqin Zhou - zkzhu@ustc.edu; Yanzhi Du - yzdu@sibs.ac.cn; Winston Patrick Kuo - winston_kuo@hsdm.harvard.edu; Ji Zhang - jizhang@sibs.ac.cn; Mier Jiang - jiangme2005@yahoo.com.cn; Jason Gang Jin* - gjin@sibs.ac.cn

* Corresponding author

Published: I August 2008

BMC Genomics 2008, 9:369

doi: $10.1|86 /| 47 \mid-2164-9-369$
Received: 7 December 2007

Accepted: I August 2008

This article is available from: http://www.biomedcentral.com/I47I-2164/9/369

(c) 2008 Fu et al; licensee BioMed Central Ltd.

This is an Open Access article distributed under the terms of the Creative Commons Attribution License (http://creativecommons.org/licenses/by/2.0), which permits unrestricted use, distribution, and reproduction in any medium, provided the original work is properly cited.

\begin{abstract}
Background: Peripheral arterial disease (PAD), a major manifestation of atherosclerosis, is associated with significant cardiovascular morbidity, limb loss and death. However, mechanisms underlying the genesis and progression of the disease are far from clear. Genome-wide gene expression profiling of clinical samples may represent an effective approach to gain relevant information.
\end{abstract}

Results: After histological classification, a total of 30 femoral artery samples, including II intermediate lesions, 14 advanced lesions and 5 normal femoral arteries, were profiled using Affymetrix microarray platform. Following real-time RT-PCR validation, different algorithms of gene selection and clustering were applied to identify differentially expressed genes. Under a stringent cutoff, i.e., a false discovery rate (FDR) $<0.5 \%$, we found 366 genes were differentially regulated in intermediate lesions and 447 in advanced lesions. Of these, 116 genes were overlapped between intermediate and advanced lesions, including 68 up-regulated genes and 48 downregulated ones. In these differentially regulated genes, immune/inflammatory genes were significantly up-regulated in different stages of PAD, (85/230 in intermediate lesions, 37/172 in advanced lesions). Through literature mining and pathway analysis using different databases such as Gene Ontology (GO), and the Kyoto Encyclopedia of Gene and Genomics (KEGG), genes involved in immune/inflammatory responses were significantly enriched in up-regulated genes at different stages of $\operatorname{PAD}(P<0.05)$, revealing a significant correlation between immune/inflammatory 
responses and disease progression. Moreover, immune-related pathways such as Toll-like receptor signaling and natural killer cell mediated cytotoxicity were particularly enriched in intermediate and advanced lesions $(P<0.05)$, highlighting their pathogenic significance during disease progression.

Conclusion: Lines of evidence revealed in this study not only support previous hypotheses, primarily based on studies of animal models and other types of arterial disease, that inflammatory responses may influence the development of PAD, but also permit the recognition of a wide spectrum of immune/inflammatory genes that can serve as signatures for disease progression in PAD. Further studies of these signature molecules may eventually allow us to develop more sophisticated protocols for pharmaceutical interventions.

\section{Background}

Peripheral arterial occlusive disease (PAD) is a major manifestation of atherosclerosis and is commonly found in elderly patients. Epidemiological studies have shown that PAD affects 8 to 10 million adults in the United States [1]. Most patients with PAD are asymptomatic. The disease is primarily diagnosed by an ankle brachial index $(\mathrm{ABI})<0.9$. The most common symptom of mild-to-moderate PAD is intermittent claudication, which is present in about one third of symptomatic patients [1]. In addition to leg symptoms, patients with PAD are at an increased risk for developing new coronary events and eventually death from cardiovascular disease. Although conventional procedures such as stents, arterectomies, angioplasty, and bypass surgery have been successful in improving clinical symptoms of PAD to a large extent [2], ultimately elimination of the disease may require sophisticated protocols of pharmaceutical interventions, which may depend on better understanding of molecular mechanisms involved in the disease.

Previous studies have implicated the involvement of the immune system in atherosclerosis formation and progression. Animal models have been used to test the contributions of components of the immune system [3,4]. Cellular involvement of macrophages was found to be important in the formation and progression of atherosclerosis in animal models [4]. In addition, various immune-related genes have been examined in an atherosclerosis animal model, and genes such as CXCR6, CXCL10, CXCR3 and CXCL16/scavenger receptor have been shown to be involved in the progression of atherosclerosis in animal models [5-8]. In humans, many immune cells such as macrophages, lymphocytes, mast cells, and $\mathrm{T}$ cells are found in atherosclerosis [9]. These findings suggest that the immune system plays important roles in atherogenesis. However, data available to date are primarily derived from studies of atherosclerosis in the coronary or/and the carotid arteries, whereas data derived from clinical samples of PAD appear to be particularly limited.

In the past decade, microarray analysis using highthroughput screening technology has emerged as an important tool to study gene expression patterns and to study molecular events in complex diseases [10-12]. In this study, Affymetrix GeneChips were used to perform gene expression profiling of femoral atherosclerotic lesions to fully characterize the peripheral arterial wall gene expression patterns associated with atherosclerosis. By statistical analysis, hundreds of known and novel genes were identified that differentially express in PAD. Genes involved in immune/inflammatory responses appeared to be significantly enriched in the set of genes up-regulated in different stages of PAD. To further examine the expression patterns of individual genes in the context of particular biological or molecular pathways, gene functional enrichment was performed using Gene Ontology and KEGG database. The results revealed that immune system-related categories and pathways were significantly overrepresented in the progression of the disease, suggesting that up-regulation of immune/ inflammatory genes may be critical components of the disease progression expression signature associated with atherosclerosis. These findings may provide new insights and foster a better understanding of the mechanism of PAD.

\section{Results \\ Patient classification and outcome}

Histological characterization of 30 collected peripheral artery samples was conducted based on the criteria of the American Heart Association. Of these samples, 15 were classified as grade III (intermediate lesions), one as grade IV and fourteen as grade $\mathrm{V}$ (advanced lesions). Among them, 11 intermediate lesions samples (grade III) and 14 advanced lesions samples (grade V) had RNA of sufficient quality and quantity for hybridization. Representative images of the different stages are shown in Figure 1. Further details of these 25 samples are listed in Table 1. As shown, there was no significant difference between the intermediate lesions and the advanced lesions group except for indications of hypertension. In the intermediate lesions group, 4 patients $(36.4 \%)$ presented with hypertension, while 9 hypertensive patients $(64.3 \%)$ were found in the advanced lesions group. 
Table I: Patient characteristics for the $\mathbf{2 5}$ samples in the microarray analysis

\begin{tabular}{|c|c|c|}
\hline & Intermediate lesions group $(\mathrm{n}=\mathrm{I} \mathrm{I})$ & Advanced lesions group $(n=14)$ \\
\hline Age* & $76.1 \pm 4.68$ & $76.6 \pm 3.79$ \\
\hline Male, n (\%) & $8(72.7)$ & $12(85.7)$ \\
\hline \multicolumn{3}{|c|}{ Hypertensiont, n (\%) } \\
\hline Yes & $4(36.4)$ & $9(64.3)$ \\
\hline no & $7(63.6)$ & $5(35.7)$ \\
\hline \multicolumn{3}{|c|}{ Hypercholesterolemia, n (\%) } \\
\hline Yes & $9(81.8)$ & II (78.6) \\
\hline no & $2(18.2)$ & $3(21.4)$ \\
\hline \multicolumn{3}{|c|}{ Diabetes, n (\%) } \\
\hline Yes & $7(63.6)$ & $10(71.4)$ \\
\hline no & $4(36.4)$ & $4(28.6)$ \\
\hline
\end{tabular}

*as mean \pm SD.

$+P<0.05$ (Student $t$ test)

\section{Differentially regulated genes in intermediate lesions} Identifying differential expression genes was achieved for different stages by using Significance Analysis of Microarrays (SAM) with a false discovery rate (FDR) of $0.5 \%$. Comparative analysis revealed that 366 genes were differentially expressed in intermediate lesions when compared

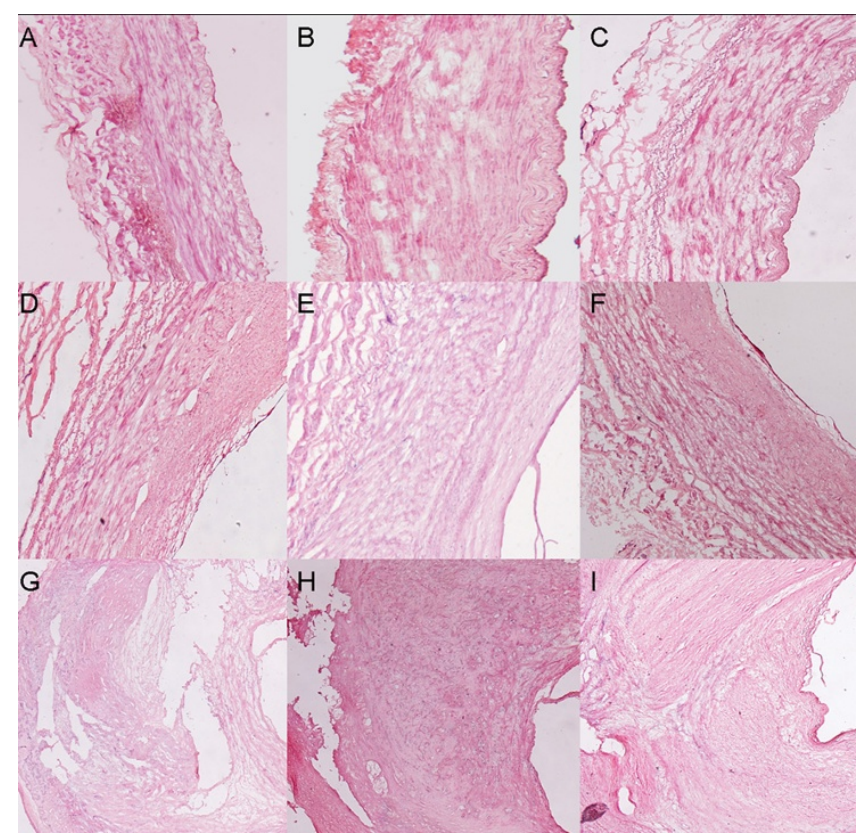

\section{Figure I}

Histological characteristics of various severities of femoral atherosclerotic lesions in PAD patients. HE stain analysis of histological characteristics of collected femoral non-atherosclerotic arteries and atherosclerotic arteries. 8 um cryostat sections were stained with hematoxylin and eosin, dehydrated in graded alcohol, and cover-slipped with permanent mounting solution after xylene clearing. Three representative samples are listed: normal artery $(A, B, C)$, intermediate lesions (D, E, F), and advanced lesions $(G, H, I)$. to normal femoral arteries, of which 230 genes were upregulated and 136 were down-regulated [see Additional file 1]. The 100 most differentially expressed genes between intermediate lesions and normal femoral arteries are shown in Figure 2A. Notably, in the up-regulated genes, up to 85 genes have been reported to be involved in immune response, such as HLA-DQB1, HLA-DRB1, CCR1, CXCR4, C1QB and TLR7 [see Additional file 2]. In addition, a large number of genes known to encode proteins crucial for proteolysis (CTSB, CTSC, CTSD and CTSS) and cell proliferation (BTG1, BTG2, CDKN1A, and MCM5) appeared to be significantly changed. Since BTG1 and BTG2 are known to be involved in anti-proliferation activities, it can be of interest to further investigate their potential roles in PAD in detail. MCM5 is heavily involved in chromosomal stability. Among the down-regulated genes, those involved in calcium signaling (CAMK2G), transport (SLC22A3, CYP1A1 and ATP5H), metabolism (GCSH and PLA2G4A), and protein amino acid dephosphorylation (PTPN20) were found to be significantly down-regulated. The Gene Ontology functional categories in which intermediate lesions are overrepresented are illustrated in Table 2 and additional data [see Additional file 3]. As shown in the table, the most significant biological process categories in the up-regulated genes are immune response, humoral immune response, inflammatory response, and $\mathrm{T}$ cell proliferation (Z-score>5). For down-regulated genes, the significant ones mainly represent metabolism and catabolism-related categories.

\section{Differentially regulated genes in advanced lesions}

When advanced lesions were compared to normal femoral arteries, 447 genes were identified, of which 172 genes were up-regulated and 275 were down-regulated [see Additional file 4]. The list of the 100 most differentially expressed genes is shown in Figure 2B. Interestingly, up to 37 genes involved in the immune system response, such as CCR1, CX3CR1, TLR1 and TLR7, were found to be up- 
Table 2: Biological process categories overrepresented in intermediate lesions

\begin{tabular}{|c|c|c|}
\hline GO Name & Z-score & $P$-value \\
\hline \multicolumn{3}{|l|}{ For up-regulated genes } \\
\hline immune response & $|4.49|$ & 0.000 \\
\hline antigen processing, endogenous antigen via $\mathrm{MHC}$ class I & 3.821 & 0.014 \\
\hline antigen processing, exogenous antigen via MHC class II & 5.968 & 0.001 \\
\hline cellular defense response & 5.669 & 0.000 \\
\hline cell-mediated immune response & 3.093 & 0.038 \\
\hline T-helper I type immune response & 3.214 & 0.035 \\
\hline humoral immune response & 9.451 & 0.000 \\
\hline complement activation & 3.608 & 0.016 \\
\hline inflammatory response & 8.515 & 0.000 \\
\hline neutrophil chemotaxis & 6.629 & 0.004 \\
\hline leukocyte adhesion & 4.767 & 0.013 \\
\hline immune cell activation & 3.268 & 0.011 \\
\hline immune cell migration & 6.25 & 0.000 \\
\hline cell surface receptor linked signal transduction & 2.082 & 0.042 \\
\hline integrin-mediated signaling pathway & 2.633 & 0.035 \\
\hline intracellular signaling cascade & 4.367 & 0.000 \\
\hline MAPKKK cascade & 2.413 & 0.037 \\
\hline myeloid cell differentiation & 2.876 & 0.042 \\
\hline lipid transport & 3.255 & 0.011 \\
\hline Phagocytosis & 4.757 & 0.005 \\
\hline Apoptosis & 2.091 & 0.049 \\
\hline cell proliferation & 4.257 & 0.000 \\
\hline $\mathrm{T}$ cell proliferation & 5.489 & 0.003 \\
\hline oxygen transport & 3.255 & 0.016 \\
\hline icosanoid metabolism & 3.524 & 0.010 \\
\hline lipoprotein metabolism & 3.291 & 0.017 \\
\hline \multicolumn{3}{|l|}{ For down-regulated genes } \\
\hline cellular catabolism & 2.708 & 0.014 \\
\hline amine catabolism & 3.599 & 0.011 \\
\hline glycine catabolism & 8.457 & 0.003 \\
\hline glycine metabolism & 5.817 & 0.008 \\
\hline ubiquitin cycle & 2.092 & 0.041 \\
\hline protein folding & 3.088 & 0.008 \\
\hline Endocytosis & 2.521 & 0.032 \\
\hline
\end{tabular}

GO analysis was applied to differentially expressed genes in intermediate lesions compared with normal controls. Similar significant categories were not included to reduce redundancy. The first half of the table indicates categories highly significant for up-regulated genes; the second half of the table shows categories highly significant for down-regulated genes. Calculated $p$-values and Z-scores for each category are shown. The corresponding cell component categories and molecular function categories are available in additional data [see Additional file 3]

regulated in advanced lesion [see Additional file 5], which might suggest that these immune/inflammatory related genes could serve as expression signatures characterizing different stages of PAD. In addition, genes constituting a major portion of the vascular extracellular matrix were significantly up-regulated in advanced lesions, including COL1A1, COL3A1, COL1A2, COL5A1, COL6A1, COL6A3 and $L A M B 1$, suggesting that these genes could be involved in the femoral artery occlusion in PAD. GO analysis further confirmed the above findings, by highlighting categories of immune response, humoral immune response, inflammatory response and I-kappaB kinase/NF-kappaB cascades (Z-score>5) (Table 3 and Additional file 6). For down-regulated genes, those involved in ion transport (GRIA2 and SLC22A3) and protein folding (DNAJB5) appeared to be the most significantly down-regulated in advanced lesions. GO analysis showed that the most significant categories for down-regulated genes were response to protein stimulus, RNA metabolism, and protein folding (Table 3 and Additional file 6).

In parallel, further data analysis revealed that many genes were over-represented in both intermediate and advanced lesions vs. normal controls. Of these genes, 68 were found to be commonly up-regulated and 48 were found to be commonly down-regulated (Figure 3 ). The list of com- 
A

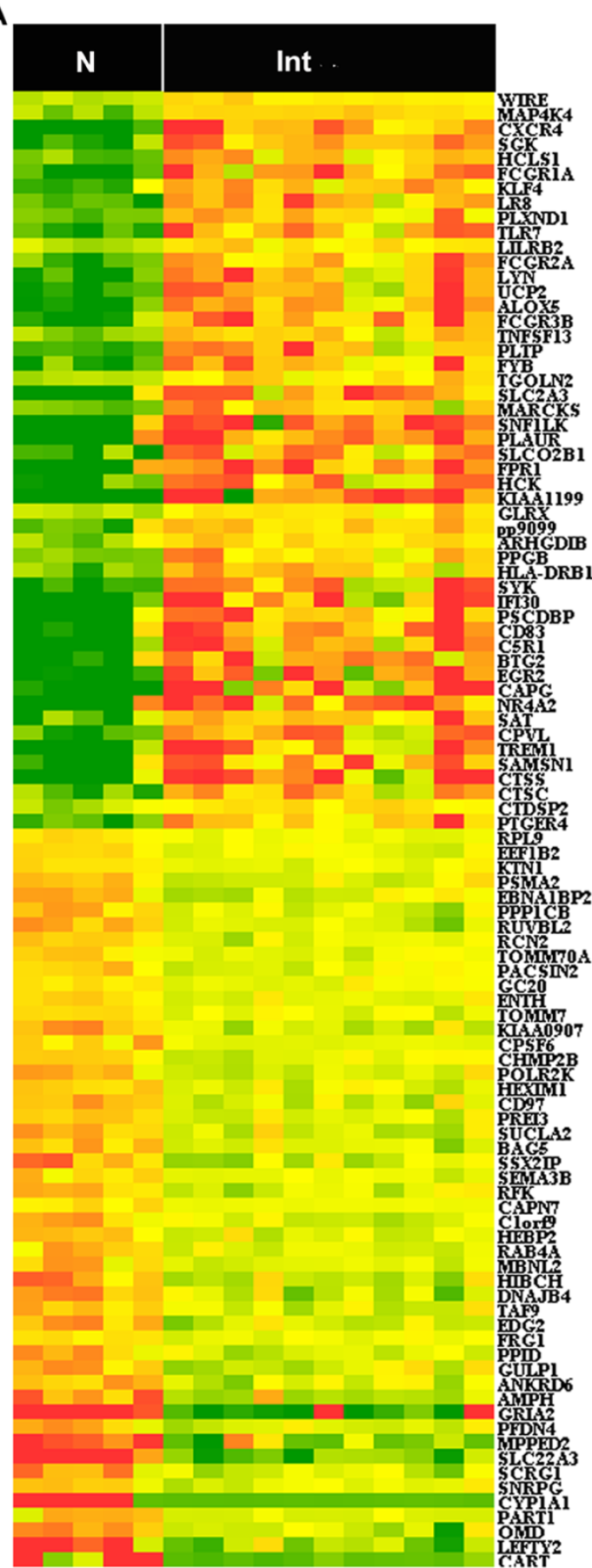

B

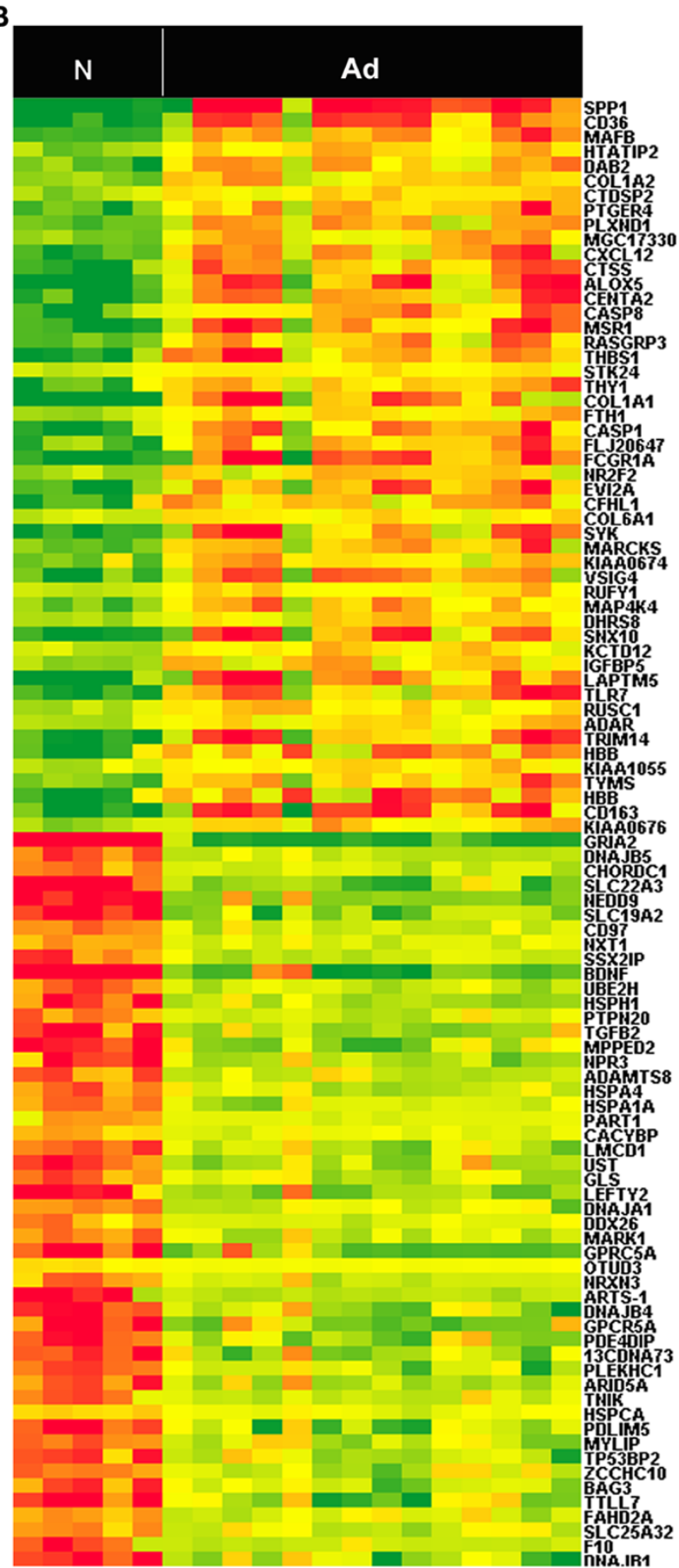

Figure 2

Heatmap of the 100 most differentially expressed genes in intermediate lesions and advanced lesions, respectively. SAM analysis reveals genes with differential expression in PAD. This analysis compared plaques from within arteries of either intermediate $(n=\mid I)$ or advanced lesions $(n=14)$ to normal control group, respectively. Heatmap representation of the 100 most differentially expressed in intermediate lesions (A) and advanced lesions (B). Samples are displayed in columns and genes in rows. Gene expression is represented as a color, normalized across each row, with brighter red for higher values and brighter green for lower values. Gene symbols are listed to the right. N (Normal control group), Int (intermediate lesions group), Ad(advanced lesions group). The list of differentially expressed genes in intermediate lesions and advanced lesions is provided [see Additional file I and Additional file 4]. 
Table 3: Biological process categories overrepresented in advanced lesions

\begin{tabular}{lrr}
\hline GO Name & Z-score & P-value \\
\hline For up-regulated genes & & \\
\hline & & \\
\hline immune response & 6.305 & 0.000 \\
cellular defense response & 2.94 & 0.026 \\
humoral immune response & 5.392 & 0.000 \\
inflammatory response & 6.179 & 0.000 \\
immune cell migration & 4.778 & 0.014 \\
intracellular signaling cascade & 4.297 & 0.001 \\
small GTPase mediated signal transduction & 2.372 & 0.024 \\
I-kappaB kinase/NF-kappaB cascade & 6.408 & 0.000 \\
T cell proliferation & 4.186 & 0.016 \\
induction of apoptosis & 3.709 & 0.007 \\
oxygen transport & 4.778 & 0.003 \\
amine transport & 3.051 & 0.024 \\
anion transport & 3.373 & 0.006 \\
phosphate transport & 6.139 & 0.000 \\
organic acid transport & 2.634 & 0.039 \\
nucleoside monophosphate metabolism & 5.853 & 0.001 \\
cell adhesion & 3.958 & 0.000
\end{tabular}

For down-regulated genes

\begin{tabular}{lrl}
\hline response to protein stimulus & 6.79 & 0.000 \\
response to unfolded protein & 6.79 & 0.000 \\
integrin-mediated signaling pathway & 4.36 & 0.003 \\
Apoptosis & 2.838 & 0.003 \\
muscle cell differentiation & 3.771 & 0.032 \\
vasculature development & 3.795 & 0.005 \\
neuron differentiation & 3.993 & 0.003 \\
vitamin transport & 3.771 & 0.023 \\
RNA metabolism & 3.476 & 0.000 \\
RNA splicing & 3.497 & 0.005 \\
glutamine metabolism & 4.901 & 0.004 \\
macromolecule metabolism & 2.214 & 0.023 \\
protein folding & 5.643 & 0.000 \\
cell-matrix adhesion & 2.605 & 0.036 \\
peptide hormone secretion & 5.401 & 0.004 \\
\hline
\end{tabular}

GO analysis was applied to differentially expressed genes in advanced lesions compared with normal controls. Some other selected categories are shown. The first half of the table indicates categories highly significant for up-regulated genes; the second half of the table shows categories highly significant for down-regulated genes. Calculated $p$-values and Z-scores for each category are shown. The corresponding cell component categories and molecular function categories are available in additional data [see Additional file 6]

monly up-regulated genes is available [see Additional file 7]. Some of these overlapping genes, such as CTSB, CCR1, ALOX5, and SPP1, have been previously reported to play important roles in atherogenesis [13-16]. Accordingly, these commonly regulated genes can therefore be important for the progression of PAD. In contrast, a much larger number of genes appear to be characteristically expressed in either intermediate lesions or advanced lesions, which may therefore serve as stage-specific signatures of PAD.

\section{Differential gene expression in disease progression}

Intermediate lesions and advanced lesions represent different stages in disease progression of PAD. Identification of genes that exhibit characteristic expression patterns in different stages may provide information relevant to the progression of PAD. For this reason, expression profiles of normal arteries, intermediate lesions and advanced lesions were analyzed by the SAM multiclass method. Out of this analysis, 614 genes appeared to be differentially expressed in the progression of PAD with a FDR $<0.5 \%$ [see Additional file 8]. Hierarchical clustering analysis suggested that the expression patterns of the genes could be assigned to three major groups (Figure 4). The first group represents those genes commonly expressed in both intermediate and advanced lesions (Cluster II). GO terms indicate that these genes are mainly involved in the immune response, inflammatory response, cellular defense and various signaling pathways (Table 4 and Additional file 9). These results further support the notion that the immune system may play an important role in the progression of PAD. The second group represents specifically down-regulated genes in advanced lesions (Cluster II). GO terms indicate that these genes are primarily involved in cell cycle, apoptosis, multicellular organism development and protein folding (Table 4 and Additional file 9). Genes in the third group are represented by those down-regulated in both intermediate lesions and advanced lesions (Cluster III). GO terms indicate that these genes are mainly involved in neurogenesis, protein modification, RNA splicing, and blood pressure regulation (Table 4 and see Additional file 9). In addition, we have performed data analysis restricted to male subjects. Up to $85 \%$ genes identified in male subjects are the same as those identified in the total samples (data not shown), which suggests that the potential gender-biases is minimal. Taken together, genes commonly up-regulated in intermediate and advanced stages are typically represented by those involved in immune and inflammatory responses, implicating enhanced immune response activities during the progression of the disease, whereas downregulated genes in the both disease stages are primarily represented by those involved in various aspects of cell proliferation and differentiation.

\section{Validation of gene transcription by real-time PCR}

Real-time PCR is still the gold standard for quantitative analysis of mRNA. In order to validate the microarray results, RT- PCR was carried out on the same set of samples that were analyzed by the microarray approach. The results were highly correlated with those from the array data. (The correlation coefficient for microarray and RTPCR was $0.835 \pm 0.076$ ). Representative RT-PCR results of 6 genes are shown in Figure 5. 


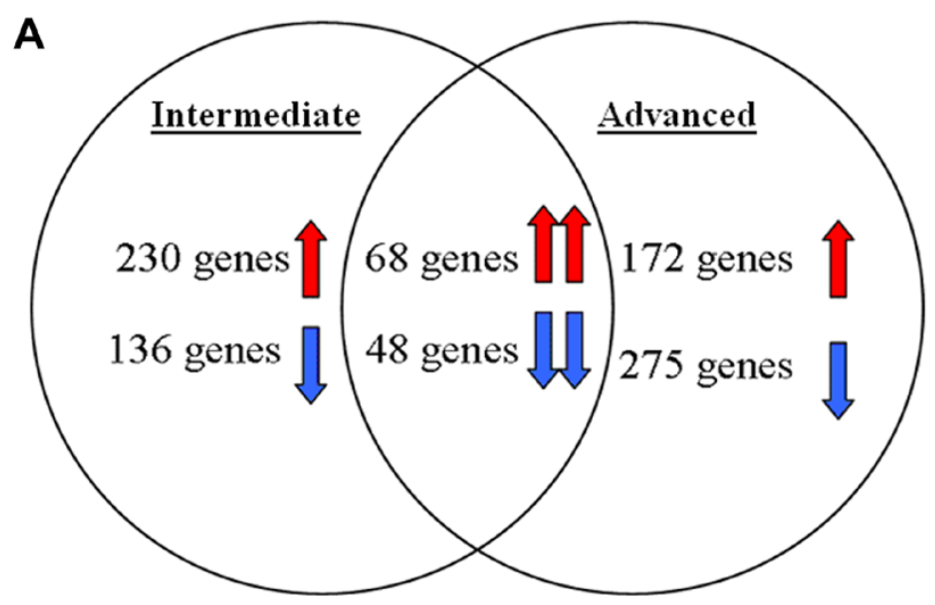

B

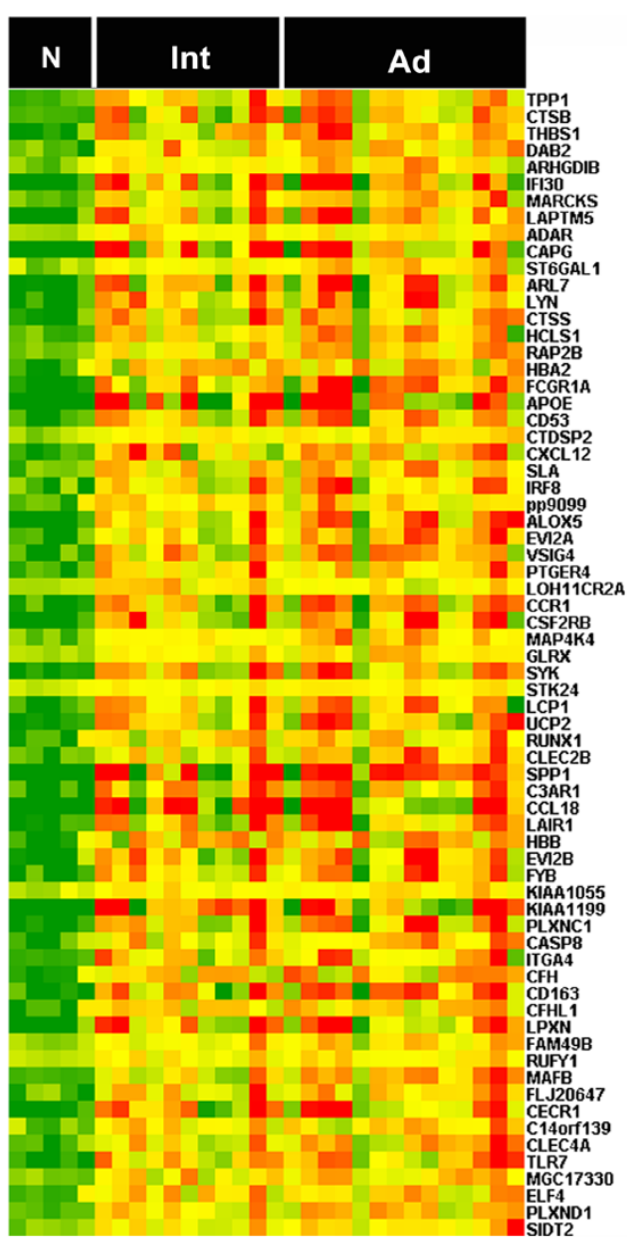

C

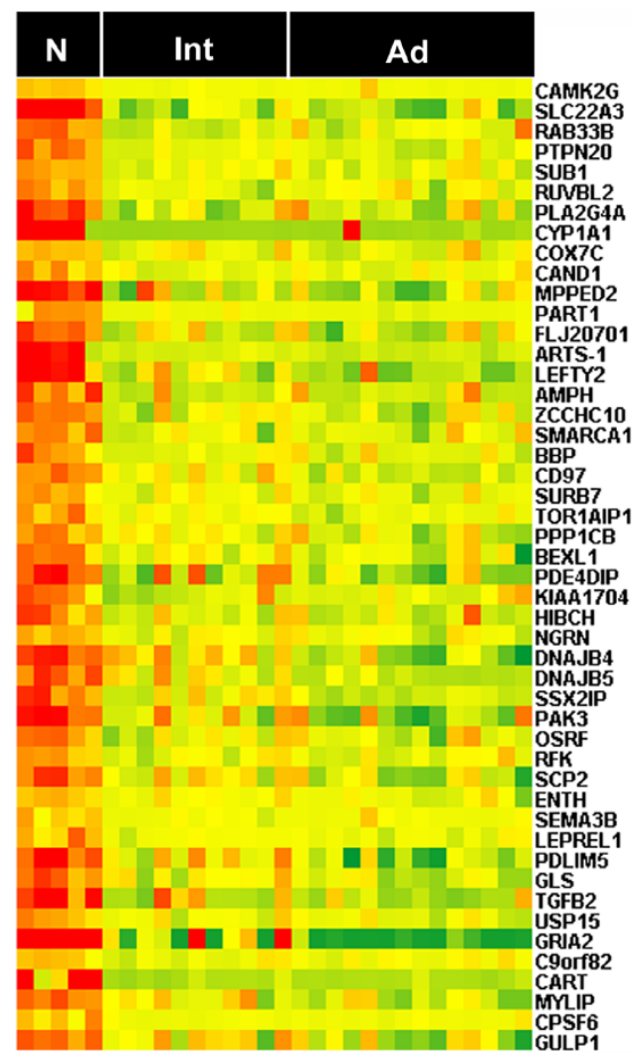

Figure 3

Over-represented genes in both intermediate lesions and advanced lesions. A. The genes whose expressions were significantly changed in intermediate lesions and advanced lesions, respectively, are shown in a Venn diagram. B, C. Heatmap representation of commonly up-regulated genes (B) and commonly down-regulated genes $(C)$ in overlapping genes, respectively. Samples are displayed in columns and genes in rows. Gene expression is represented as a color, normalized across each row, with brighter red for higher values and brighter green for lower values. Gene symbols are listed to the right. $\mathrm{N}$ (Normal control group), Int (intermediate lesions group), Ad (advanced lesions group). 
Table 4: GO discovered categories for disease progression manner analysis

\begin{tabular}{|c|c|c|}
\hline GO Name & Z-Score & P-value \\
\hline \multicolumn{3}{|l|}{ For Cluster I } \\
\hline immune response & 8.908 & 0.000 \\
\hline inflammatory response & 7.643 & 0.000 \\
\hline cellular defense response & 5.938 & 0.001 \\
\hline chemotaxis & 7.611 & 0.000 \\
\hline cell surface receptor linked signal transduction & 2.937 & 0.009 \\
\hline cytokine and chemokine mediated signaling pathway & 4.378 & 0.004 \\
\hline JAK-STAT cascade & 3.254 & 0.020 \\
\hline MAPKKK cascade & 3.062 & 0.006 \\
\hline endocytosis & 1.956 & 0.049 \\
\hline angiogenesis & 4.452 & 0.000 \\
\hline cell adhesion & 4.580 & 0.000 \\
\hline ion homeostasis & 3.090 & 0.010 \\
\hline
\end{tabular}

\section{For Cluster II}

\begin{tabular}{|c|c|c|}
\hline apoptosis & 3.871 & 0.002 \\
\hline cell cycle & 4.108 & 0.000 \\
\hline cell growth & 2.285 & 0.037 \\
\hline epidermal cell differentiation & 3.075 & 0.044 \\
\hline protein folding & 3.677 & 0.001 \\
\hline macromolecule metabolic process & 3.022 & 0.005 \\
\hline RNA metabolic process & 5.378 & 0.000 \\
\hline transcription & 4.593 & 0.000 \\
\hline peripheral nervous system development & 2.950 & 0.046 \\
\hline multicellular organismal development & 3.927 & 0.000 \\
\hline vitamin transport & 4.426 & 0.008 \\
\hline response to stimulus & 4.579 & 0.000 \\
\hline circadian rhythm & 5.090 & 0.001 \\
\hline \multicolumn{3}{|l|}{ For Cluster III } \\
\hline neurogenesis & 2.612 & 0.014 \\
\hline neuron recognition & 3.315 & 0.046 \\
\hline cell motility & 2.093 & 0.042 \\
\hline glycogen metabolic process & 2.999 & 0.025 \\
\hline protein modification process & 3.166 & 0.001 \\
\hline RNA processing & 2.183 & 0.042 \\
\hline RNA splicing & 2.591 & 0.021 \\
\hline phosphate metabolic process & 2.346 & 0.024 \\
\hline blood pressure regulation & 4.028 & 0.008 \\
\hline heart contraction & 2.901 & 0.022 \\
\hline
\end{tabular}

GO analysis was applied to differentially expressed genes in each cluster group. Some selected categories are shown. The Calculated $p$-values and Z-scores for each category are shown. The detailed GO results are provided in the additional data [see Additional file 9].

\section{Transcription factors enrichment analysis}

Transcription factors appear to play important roles in the development or progression of atherosclerosis $[17,18]$. To address whether specific transcription factors are involved in the regulation of genes associated with the progression of PAD, we conducted a transcription factor binding site enrichment study by analyzing cross-species conserved binding sites in promoter regions of genes differentially regulated during progression of PAD. Through the Fisher
Exact test, binding sites of transcription factor AP-1 and CREB appeared to be significantly enriched (q-value < 0.05). AP-1 is a transcription factor known to be involved in various cellular processes. In atherosclerosis, it has been reported in gene regulation of microphages, vascular smooth muscle cells and epithelial cells $[19,20]$. In disease progression, AP-1 was enriched to regulate expression of 72 genes (Figure 6A and Additional file 10). The enrichment of AP1 binding sites in regulated genes associ- 


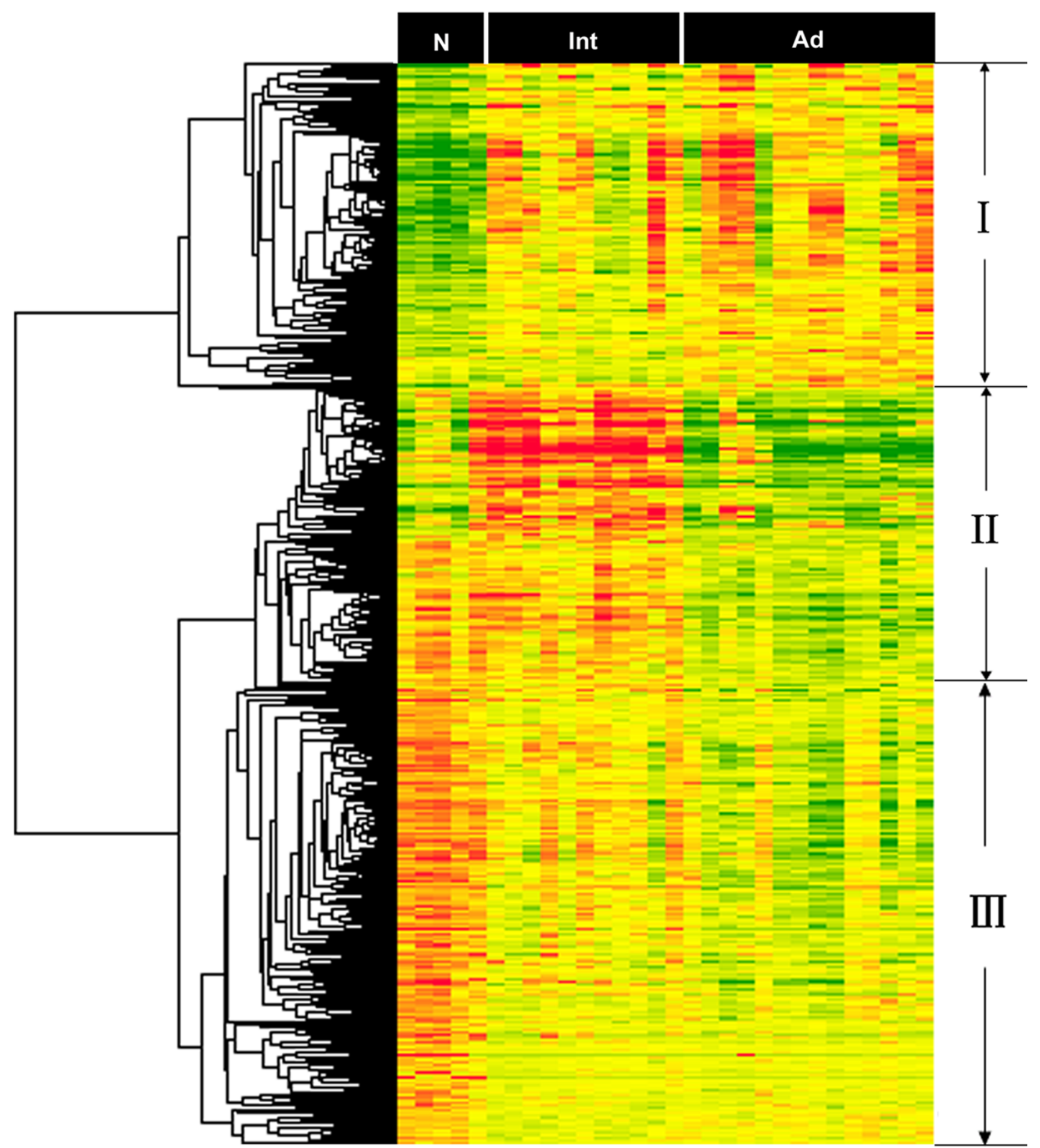

Figure 4

Hierarchical clustering analysis of the differentially expressed genes in disease progression. The differentially expressed genes analyzed by hierarchical clustering method in disease progression. The genes were classified into three major clusters by visual inspection. Clustering method: Average linking; Similarity measure: Euclidean distance. Samples are displayed in columns and genes in rows. Gene expression is represented as a color, normalized across each row, with brighter red for higher values and brighter green for lower values. N (Normal control group), Int (intermediate lesions group), Ad (advanced lesions group). The list of differentially expressed genes in disease progression is provided [see Additional file 8]. 

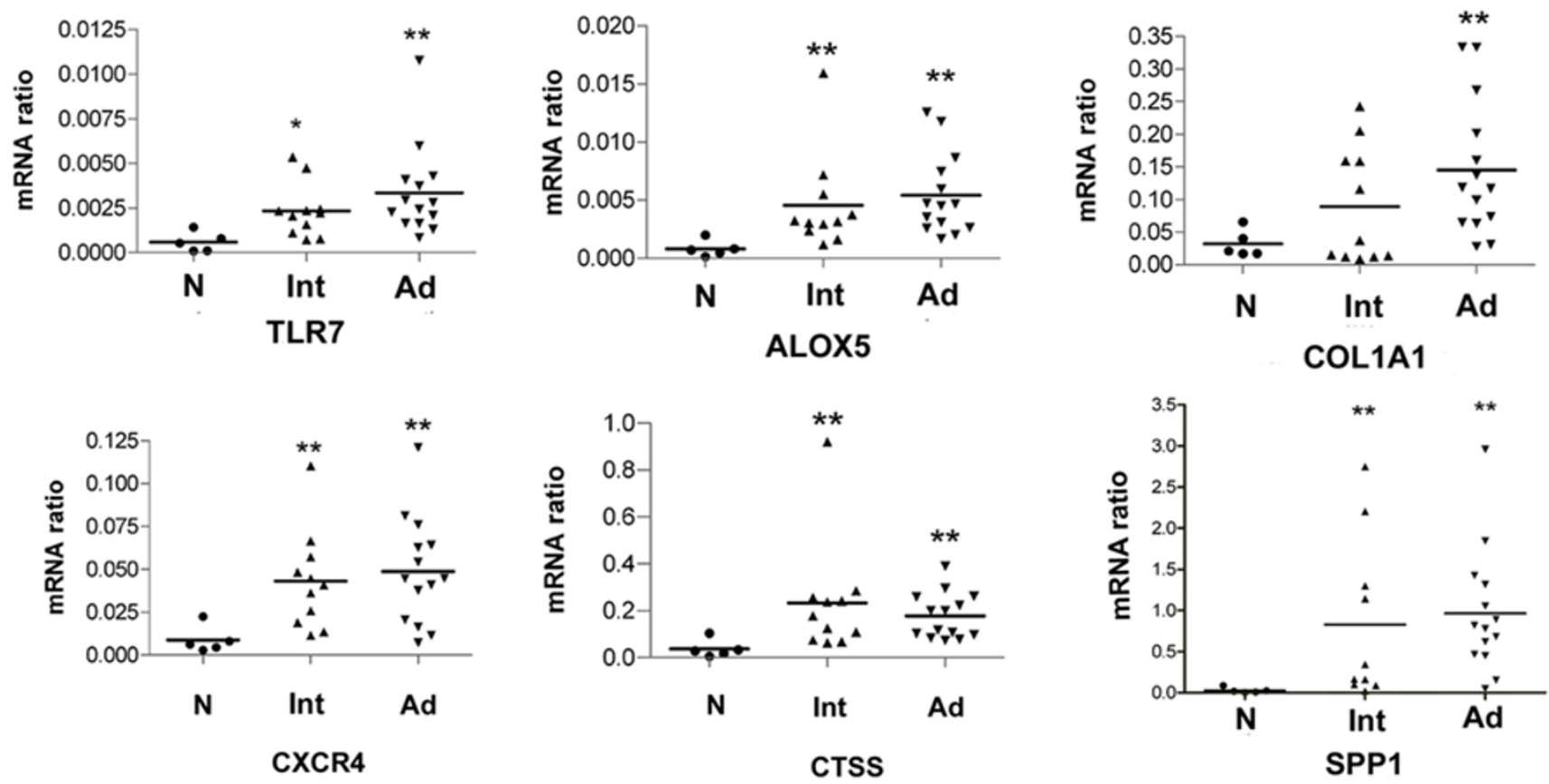

Figure 5

Real-time PCR and the relative expression level of six genes. Six genes mRNA in normal femoral artery (N, black round), intermediate lesions (Int, ascending triangle) and advanced lesions (Ad, descending triangle) were determined by realtime PCR and presented as a ratio to GAPDH mRNA. mRNA abundance in intermediate lesions or advanced lesions was differentially expressed $(* P<0.05$, and $* * P<0.0$ I, respectively) when normal samples were used for comparison.
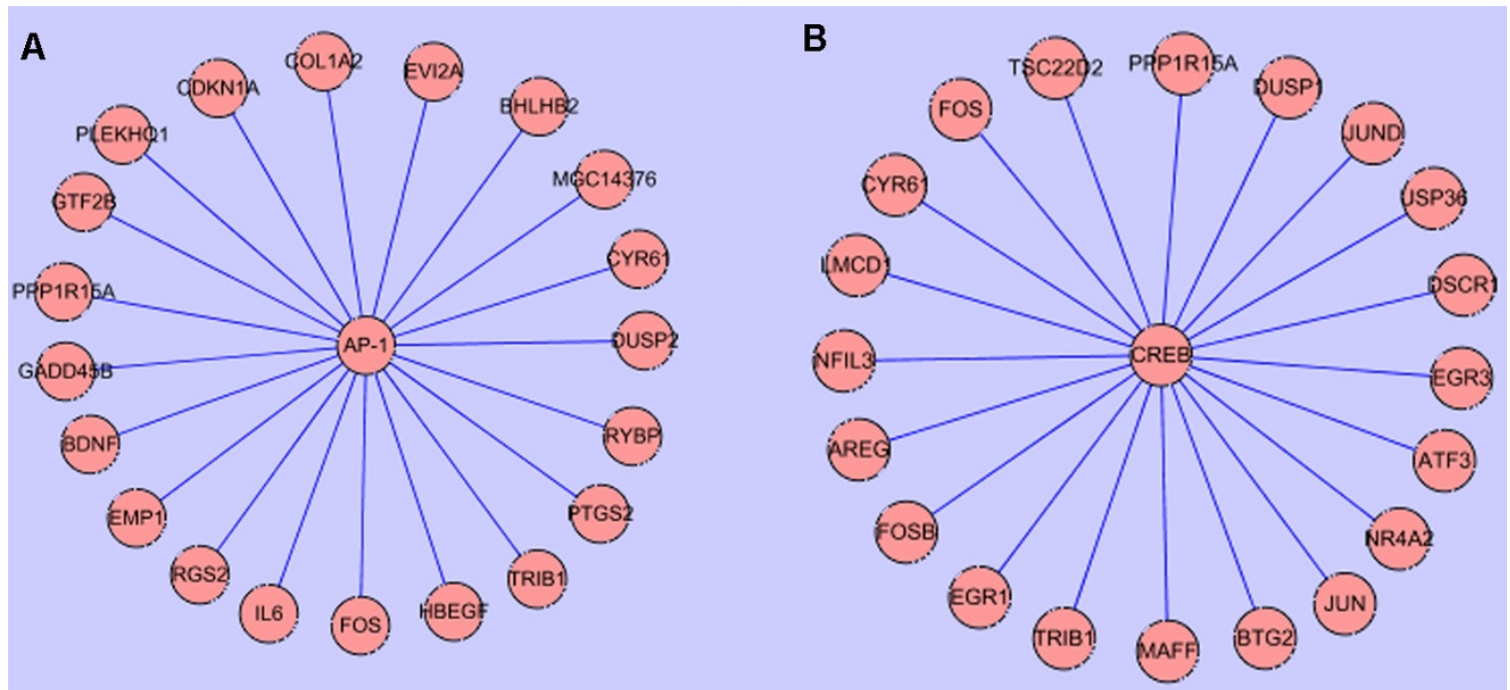

Figure 6

Enriched transcription factors and their putative target genes in disease progression. Putative targets of transcription factors were curated based on results by Xiaohui Xie. Fisher Exact test showed that two transcription factors (AP-I and CREB) were significantly enriched in disease progression (q-value $<0.05$ ). AP-I and CREB were enriched to regulate 72 and 55 genes expression, respectively. A, B. The top 20 putative target genes of AP-I and CREB were listed, respectively. A list of the enriched transcription factor and their putative targets is provided [see Additional file 10 and Additional file I I]. 
Table 5: KEGG biological pathways for differentially expressed genes in different stages

\begin{tabular}{|c|c|c|c|}
\hline KEGG Pathway Name & Pathway ID & Genes involved & P-Value \\
\hline \multicolumn{4}{|l|}{ For intermediate lesions } \\
\hline Type I Diabetes Mellitus(TIDM) & Hsa04940 & 9 & 0.002 \\
\hline Antigen Processing and Presentation(APP) & $\mathrm{Hsa04612}$ & 13 & 0.003 \\
\hline Complement and Coagulation Cascades(CCC) & Hsa046I0 & 12 & 0.003 \\
\hline Cell Adhesion Molecules(CAM) & Hsa045I4 & 17 & 0.005 \\
\hline Toll-Like Receptor Signaling Pathway(TLR) & $\mathrm{Hsa04620}$ & 13 & 0.013 \\
\hline Natural Killer Cell Mediated Cytotoxicity(NK) & $\mathrm{Hsa04650}$ & 15 & 0.029 \\
\hline \multicolumn{4}{|l|}{ For advanced lesions } \\
\hline Focal Adhesion(FA) & $\mathrm{Hsa045I0}$ & 25 & 0.000 \\
\hline ECM-Receptor Interaction(ECM) & $\mathrm{Hsa045I2}$ & 15 & 0.000 \\
\hline Toll-Like Receptor Signaling Pathway(TLR) & $\mathrm{Hsa} 04620$ & 13 & 0.002 \\
\hline Regulation of Actin Cytoskeleton(RAC) & Hsa048I0 & 20 & 0.005 \\
\hline Fc Epsilon RI Signaling Pathway(FER) & $\mathrm{Hsa04664}$ & 10 & 0.012 \\
\hline MAPK Signaling Pathway(MAPK) & $\mathrm{Hsa040I0}$ & 22 & 0.019 \\
\hline Natural Killer Cell Mediated Cytotoxicity(NK) & $\mathrm{Hsa04650}$ & 13 & 0.021 \\
\hline Long-Term Potentiation(LTP) & $\mathrm{Hsa04720}$ & 8 & 0.030 \\
\hline Cell Communication(CC) & $\mathrm{Hsa0I430}$ & 12 & 0.032 \\
\hline B Cell Receptor Signaling Pathway(BCR) & $\mathrm{Hsa04662}$ & 8 & 0.042 \\
\hline
\end{tabular}

Genes identified from SAM under FDR $<1 \%$ were tested for overrepresentation within the pathways for the KEGG under the modified Fisher Exact test assumption; $p$-Value and genes involved in the pathway are provided for each pathway

ated with PAD progression may therefore suggest an important role played by this transcription factor in the development of PAD. Through literature mining, indeed, some of the potential targets of AP1 appear to be previously reported as target genes of AP-1 [21-24]. CREB is a member of the leucine zipper family of DNA binding proteins. This transcription factor binds as a homodimer to the cAMP-responsive element and induces transcription of genes in response to hormonal stimulation [25]. A total of 55 genes were recognized as potential targets of CREB (Figure 6B and Additional file 11). Although further studies are need to elucidate detailed roles played by AP1 and CREB in PAD progression, significantly enriched binding sites and highly correlated with signature genes of PAD progression suggest that these two transcription factors may play critical roles in the development of PAD.

\section{Pathways identification by overabundant genes}

A pathway analysis database, KEGG, was then applied to genes differentially regulated in intermediate and advanced lesions. Several overrepresented pathways were identified, and the enriched pathways appeared not to be independent of one another, many genes involved in one pathway could be also involved in another pathway. This interaction is illustrated in Figure 7, and pathway abbreviations can be found in Table 5. As demonstrated, many immune-related pathways were significantly over-represented in intermediate and/or advanced lesions including TLR, NK, BCR, FER, APP, CCC and LTEM pathways. These findings, on the one hand, provide evidence supporting previous hypotheses that immune/inflammatory responses play important roles in the development of PAD, and on the other hand, demonstrate that particular components of immune/inflammatory systems can be crucial for the genesis and progression of PAD. For instance, TLR and NK pathways are shown to be particularly overrepresented in both intermediate lesions and advanced lesions, highlighting their functional importance in the disease. The TLR pathway is shown in Figure 8 with the differentially regulated genes indicated.

\section{Protein validation of TLR7 expression}

Members of the Toll receptor family are key mediators of innate immunity. They respond to various pathogen-associated stimuli and transduce complex signaling responses that are required for inflammation and for the subsequent development of adaptive immunity [26]. In atherosclerosis, TLR-mediated signaling cascades are observed in macrophages, mast cells and endothelial cells $[27,28]$. Data shown in this setting demonstrate that genes involved in TLR-mediated pathway are significantly up-regulated in intermediate or advanced lesions, including TLR1, TLR2, TLR7, and MyD88. TLR1 and TLR2 have been previously reported to be significantly regulated in atherosclerosis and their functional roles have been widely investigated in atherosclerosis $[29,30]$. However, the expression of TLR7 in atherosclerosis has not been reported before. TLR7 mediates innate responses by recognizing oligonu- 

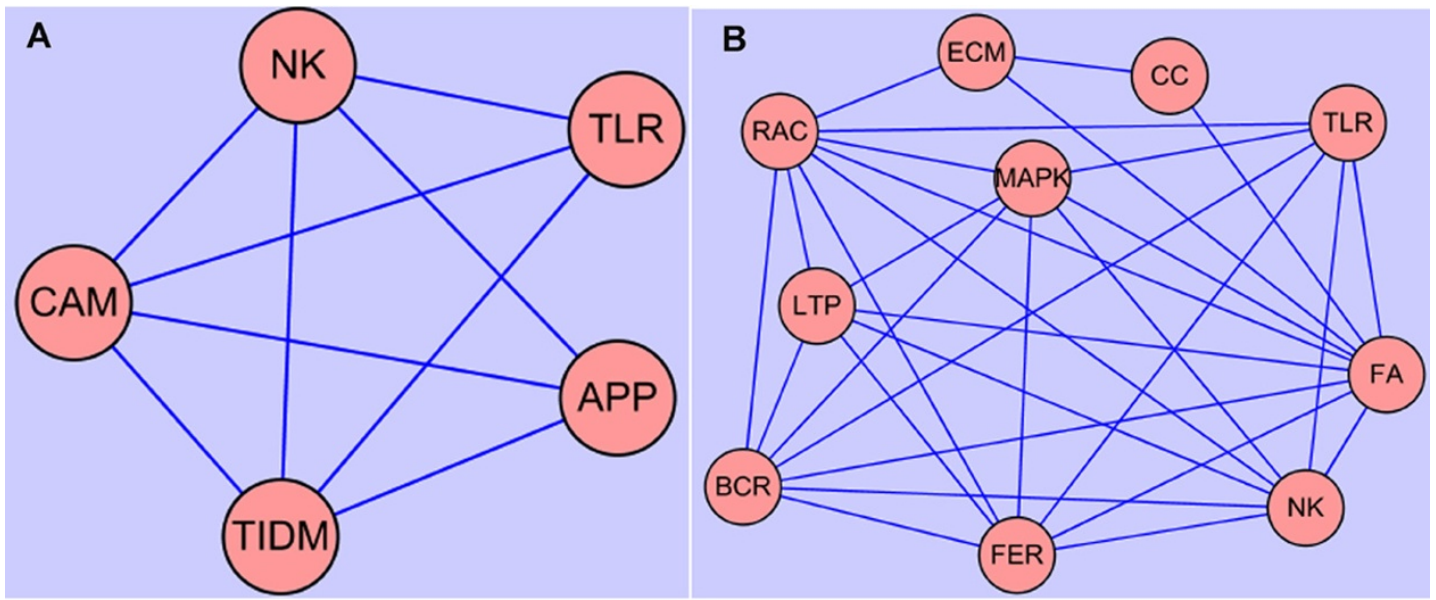

Figure 7

Interactions of KEGG pathways for differentially expressed genes in different stages of PAD. Pathways are enriched in intermediate lesions (A) and advanced lesions (B), respectively. Many genes involved in one pathway could also be involved in another pathway. A, B. Networking displayed the interaction of pathways in intermediate lesions and advanced lesions, respectively.

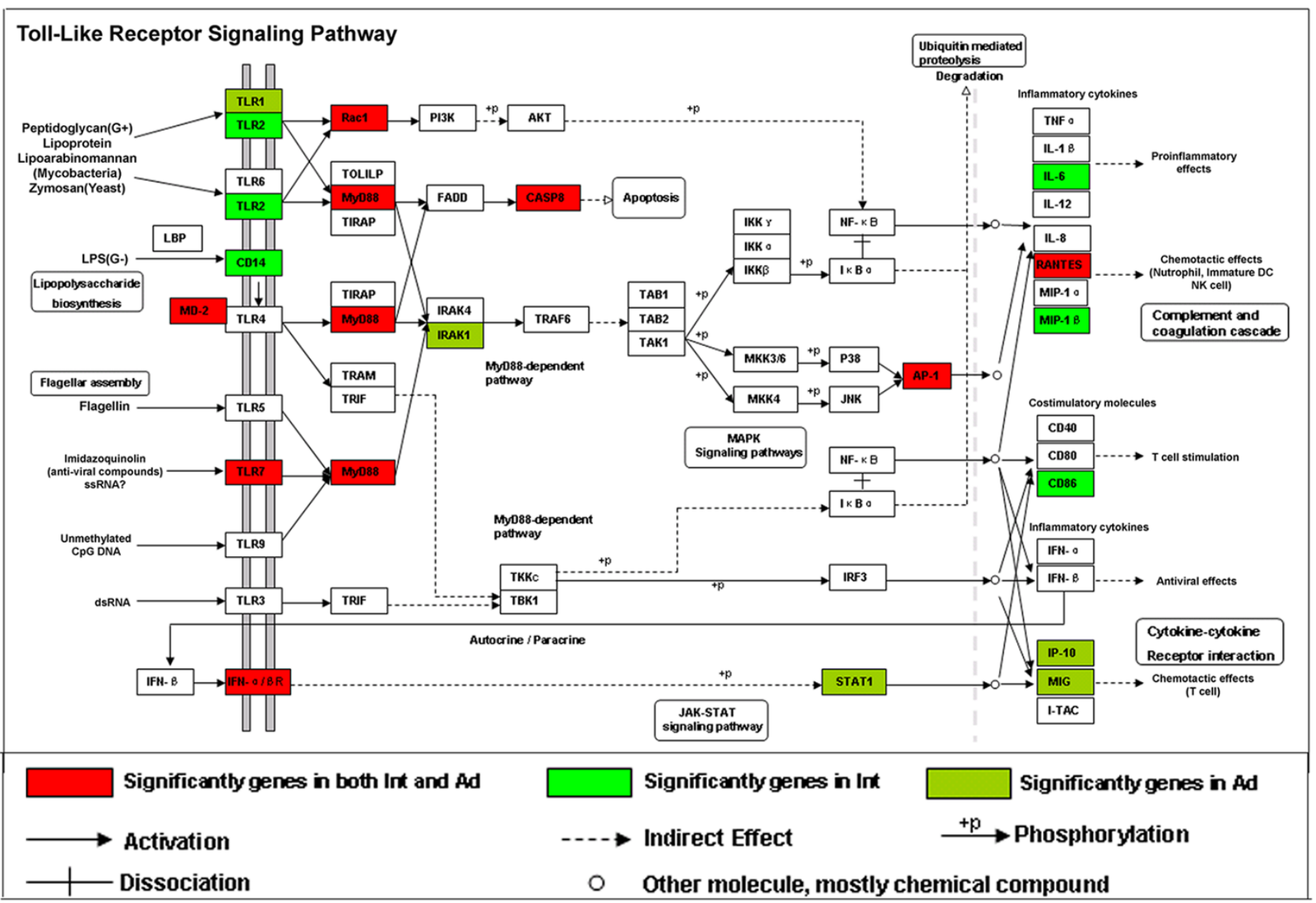

Figure 8

Over-representation of Toll-like receptor signaling pathway genes. Analysis of over-representation of differentially expressed genes in pathway from KEGG. The Toll-like receptor signaling pathway is illustrated with significantly regulated genes highlighted. 


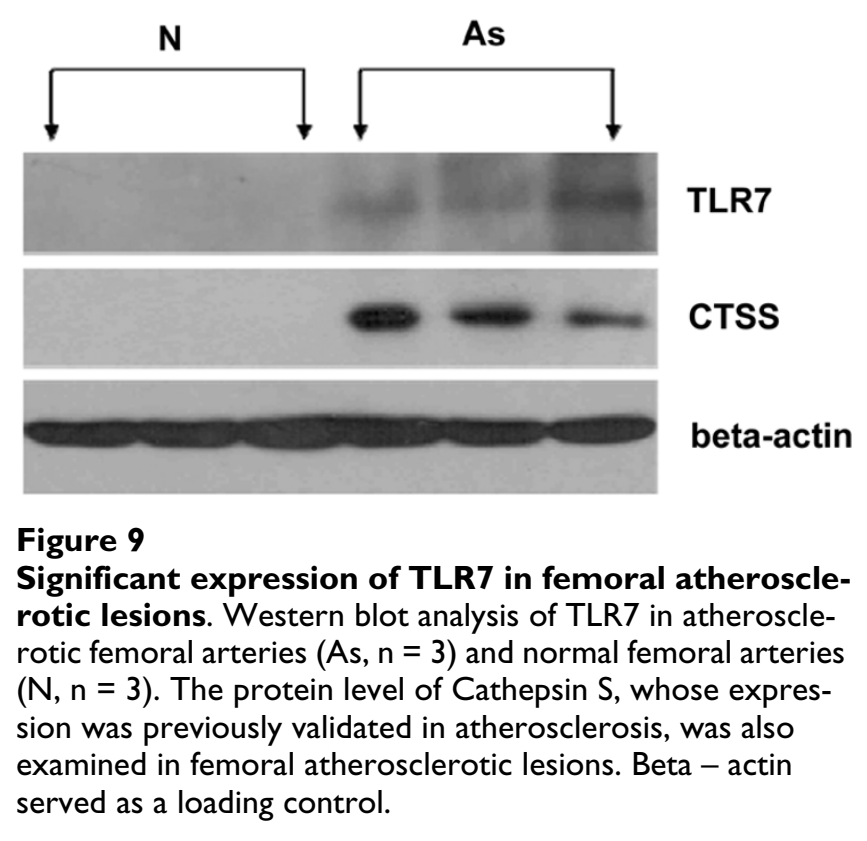

cleotide based (RNA-) molecular patterns in endocytic compartments. Our data show that it is significantly upregulated in both intermediate and advanced lesions. Western-blot analysis was performed to further validate its expression on the protein level (Figure 9). The function of TLR7 in atherogenesis is currently under further investigation.

\section{Discussion}

In the present study, we first examined the gene expression profiles of PAD. Data analysis identified a number of genes that might be significantly correlated with different levels of PAD severity. The list of differentially expressed genes in intermediate and advanced lesions contains many genes which can be important for atherosclerosis. Most of these genes have not been reported to be related to atherosclerosis before. For example, MAP4K4 is a member of the serine/threonine protein kinase family. It has been shown to specifically activate MAPK8/JNK and mediate the TNF-alpha signaling pathway [31,32]. In this study, it was significantly and consistently up-regulated in both intermediate and advanced lesions.

A large multidisciplinary study is currently underway to comprehensively assess PAD at multiple levels [33], The goal of that study is to investigate 300 symptomatic patients with PAD undergoing medical management with or without vascular intervention by lower extremity angioplasty/stenting or vein graft bypass, and to test the hypothesis that the systemic inflammatory response after vascular intervention influences the local milieu responsible for vascular repair and adaptation [33]. Identification of genes through the work may be significant in the selection of candidate genes that can be investigated through these cases-control genetic epidemiology studies. Our research supports the idea that immune responses play a key role in the development of PAD.

In this report, immune related genes were shown to be significantly expressed during the development of PAD. Gene functional analysis further revealed that immune related categories and pathways were significant enriched in the different stages of PAD. In these immune related genes, several genes have been shown to modulate the development of atherosclerosis in mice models. For example, IgG Fc receptors (FcgammaRs) play a role in activating the immune system and in maintaining peripheral tolerance. Previous research suggested that Fcy receptor deficiency protects against atherosclerosis in ApolipoproteinE knockout mice [34]. The results suggest that broadrange inhibitors of immune and inflammatory responses can be considered as potential targets for the treatment of PAD. However, gene expression patterns of immune related genes can be different in different stages of PAD. For example, in intermediate lesions, MHC class II molecules were significantly up-regulated including HLADMA, HLA-DMB, HLA-DPB1, HLA-DQB1, HLA-DRA, HLA-DRB1 and HLA-DRB5. MHC class II molecules are normally restricted to a subset of antigen presenting dendritic cells, B cells, macrophages, and thymic epithelium cells [35]. These cells can be detected close to CD4+ T cells and present peptides to the $T$ cells. The results suggest that there can be an ongoing immune activation in the intermediate lesions. However, MHC class II molecules were not differentially expressed in advanced lesions, even with a higher false discovery rate, which may suggest that the HLA-mediated immune activation may occur mainly in the progression stages of PAD. In addition, complement molecules were also significantly up-regulated in intermediate lesions, not in advanced lesions. Previous studies have implicated that activation of the complement system is probably associated with the initiation and progression of atherosclerosis $[36,37]$. Our data thus provide direct evidence from clinical samples demonstrating that complement system mainly play a role in the development stages of PAD. It is therefore conceivable that different and complex immune/inflammatory responses may take place at different stages of PAD.

Atherosclerosis is a systemic, multifocal disease leading to various symptoms and clinical events including cardiovascular disease, cerebrovascular disease, and peripheral arterial disease. Our results reveal that many genes identified in the report are also expressed in coronary or carotid atherosclerotic lesions. For example, C3AR1 and C5R1 are receptors of $\mathrm{C} 3(\mathrm{C} 3 \mathrm{a})$ and $\mathrm{C} 5 \mathrm{a}$ respectively. A recent study shows that C3AR1 and C5R1 are expressed in human 
atherosclerotic coronary plaques [38]. Double immunofluorescence staining has shown that the plaque of cells that express both $C 3 a R$ and $C 5 a R$ are macrophages, $\mathrm{T}$ cells, endothelial cells, and sub-endothelial smooth muscle cells. In addition, gene expression changes between atherosclerosis from coronary and carotid artery samples have been measured by microarray technology in recent years. One study using microarray found that 82 genes were differentially expressed in both animal model and human coronary artery atherosclerosis disease [39]. Our data confirmed 29 genes and 18 genes had significantly different expression in intermediate lesions and advanced lesions, respectively. Moreover, these genes had expression trends similar to the ones found in our data, but our data showed higher fold-changes. In these overlapping genes, 14 were reported to be involved in immune response. Another microarray study found that 206 genes were differentially expressed in aortic atherosclerosis samples [40]. Our data confirms 43 genes and 32 genes had significantly different expression in intermediate and advanced lesions $(\mathrm{FDR}<1 \%)$, respectively. Importantly, in these overlapping genes, 15 were reported to be involved in immune response. Taken together, the results suggested that immune response is a common feature in atherosclerosis-related diseases. Our microarray study differs from prior microarray studies in the array type, sample type, sample classification, and analytical techniques. Nevertheless, the high level of overlapping genes suggests that there are similar molecular mechanisms in the development of peripheral arterial disease and other atherosclerosis-related diseases.

Several limitations of our approach should be noted. First, hybridization-based microarrays, despite their immense potential, have inherent shortcomings related to deficient standardization of methods employed in normalization, statistical analysis, and so on $[41,42]$. In this study, we have attempted to limit these shortcomings by selecting subjects who were phenotypically similar to each other except for hypertension. In addition, the initial phases of data analysis, we used different normalization and statistical methods to identify differentially expressed genes. After choosing SAM, we used a rigorous false discovery rate to minimize false positive results. Expression patterns were validated by confirming mRNA expression patterns with conventional molecular techniques. We attempted, based on current literature, to suggest a potential functional role for genes whose expression was markedly altered. Second, atherosclerosis is a slow, progressive disease that may start in childhood; entirely normal arteries can only be obtained from young donors, a factor that can affect gene expression measurements. Although previous research and our data analysis suggest that age had very little effect on genes, further work is needed to identify agerelated genes. Third, the relatively small number of patients did not allow us to assess serial changes in the disease development in more detail as would have been possible in animal models [36]. Furthermore, we do not know to what extent the observed changes in gene expression translate into protein synthesis and function, and which genes cause atherosclerosis. Future studies are needed to address these issues.

\section{Conclusion}

We first examined the gene expression profiles of PAD; the results from this analysis provide an initial step towards a better understanding of molecular mechanisms underlying PAD development. Differences in immune-related responses were observable at the gene expression level. These findings may be significant for understanding the molecular basis of PAD and investigating pharmacological approaches for the prevention and amelioration of atherosclerosis in PAD.

\section{Methods \\ Tissue Harvest}

After obtaining informed consent, primary femoral artery specimens containing atherosclerotic lesions were taken from 30 patients undergoing surgical bypass or limb amputation at Shanghai Ninth People's Hospital. The specimens were immediately rinsed once with PBS and cut longitudinally by the surgeon. Three quarters of the samples were stored at once in $-80^{\circ} \mathrm{C}$ for subsequent total RNA extraction. The remaining samples were embedded in OCT medium and snap frozen for further morphological analysis. Clinical patient parameters were also registered. For controls, five normal femoral arteries were obtained from healthy donors during organ transplantation (male, mean 31.6 years; range $22-45$ years). These five samples were without clinical or gross macroscopic signs of atherosclerotic disease. The Local Ethical Committee approved all procedures in this investigation, and proper protocol was followed throughout the entire course of the experiment.

\section{Histology}

For each sample, cryostat sections of 8 um were stained with hematoxylin for $10 \mathrm{~min}$ and eosin for $2 \mathrm{~min}$, dehydrated in graded alcohol, and cover-slipped with permanent mounting solution after xylene clearing.

\section{RNA Isolation and Quantification}

Total RNA was isolated from the samples using a Trizol reagent (Invitrogen, Carlsbad, CA) and cleaned up using RNeasy Micro Kit (Qiagen, Valencia, CA) techniques. In brief, for each tissue, at least $100 \mathrm{mg}$ sample was pulverized under liquid nitrogen. After complete disruption of the tissues, the Trizol reagent was added in the amount of $1 \mathrm{ml} / 100 \mathrm{mg}$. Total RNA was extracted using the protocol supplied with the Trizol reagent. After isolation, the RNA 
was cleaned up using the RNeasy Micro Kit. To remove any contaminating genomic DNA, a DNase step was included, following the manufacturer's protocol. The RNA quantity and quality were determined by an Agilent Bioanalyzer 2100 and an Eppendorf Biophotometer. Any RNA samples that showed degradation was excluded from the study.

\section{Microarray Experiment}

One microgram of total RNA was used for generating biotin labeled cRNA. The labeling reaction was performed according to the standard Affymetrix ${ }^{\circledR}$ protocol to generate a biotin-labeled cRNA probe. The samples were hybridized to the Affymetrix ${ }^{\circledast}$ Human Genome -U133A Genechip, stained, washed and scanned according to the standard Affymetrix ${ }^{\circledR}$ protocol. The computer data files to be used in data analysis (*.dat, *.cel, *.chp) were generated with the Affymetrix GeneChip Operating Software (GCOS) Version 1.4 (Affymetrix ${ }^{\circledR}$ ), using the statistical algorithm provided. All chip samples were scanned using the same instrument and followed the same protocol. Data quality assessment was then performed following the guidance in Affymetrix data analysis fundamentals manual. All quality control results met Affymetrix recommended criteria.

\section{Data process and analysis}

The probe level intensity data were transferred to ArrayAssist $^{\circledast}$ Software (StrataGene; La Jolla, CA) for further analysis. For comparison of differential gene expression between different stage groups, the background was removed and data were normalized in accordance to the GC-RMA method [43]. GC-RMA takes into account the GC content of the probe sequences when comparing the expression intensities of the different probesets. Then, the processed gene expression data were transformed into log base 2 and filtered to delete the genes whose detection calls were "absent" in all samples.

Microarray data analysis was carried out to identify individual genes that were significantly expressed between classes by the software package SAM (please see Availability \& requirements for more information), using $\Delta=0.5$. Results from the difference analysis were clustered and displayed using the Cluster3.0 and Treeview1.1.0 software (please see Availability \& requirements for more information). Each list of differentially expressed genes was analyzed in the context of Gene Ontology (GO) in order to identify groups of genes with similar functions, or processed using MAPPFinder (Gene MicroArray Pathway Profiler; please see Availability \& requirements for more information). For each gene ontology term, the probability values were computed based on a hypergeometric distribution test by comparing (a) the number of genes annotated by the gene ontology term in a given list of dif- ferentially expressed genes with (b) the expected number of such genes. $Z$-score $>0$ and $p$-values $<0.05$ were considered significant categories.

Similar methods were used to identify curated pathways that were significantly over-represented in the data using KEGG database by using DAVID (please see Availability \& requirements for more information). For each pathway, the probability values were computed based on a modified Fisher exact test. EASE $p$-values $<0.05$ were considered significant categories. The enriched pathways are not entirely separate from one another. For example, many genes involved in MAPK signaling pathway can also be involved in other pathways, such as NK pathway. The interconnectedness information was manually extracted from the pathway. Because the nature and complexity of these interactions varied from pathway to pathway, a simple line connecting two pathways was used to represent their interaction. The interaction map was generated for the interaction of enriched pathways using CytoScape software.

Transcription factor enrichment analysis was also performed. The putative targets of transcription factors from TRANSFAC (v7.4) were discovered by Xie et al [44] and downloaded from the supplementary web site (please see Availability \& requirements for more information). All the RefSeq IDs were converted to Entrez Gene ID according to the mapping table downloaded from NCBI web site (please see Availability \& requirements for more information). Enrichment of transcription factor targets was performed as described previously [45]. The interaction map was generated for the interaction of enriched transcription factors and their putative target genes using CytoScape software

\section{Real-time QPCR Analysis}

One microgram of total RNA was reverse transcripted using random hexamers and superscript -II reverse transcriptase (Invitrogen, Carlsbad, CA). QPCR was performed by using ABI prism 7900 (ABI, Foster City, CA) and SYBR Green Detection (Toyobo, Japan). Primers were designed by using the Primer Express 2.0 software and verified by using a BLAST search. Sequences of the primers are listed [see Additional file 12]. The experimental conditions followed the manufacturer's protocol and the data were analyzed with sequence Detection Software 2.0 (ABI, Foster City, CA). Relative expression of mRNA was calculated with the comparative CT method. To standardize the amount of input RNA, the GAPDH gene was included. For each sample, the experiment was performed in triplicate.

\section{Western Blotting}

Proteins were extracted after RNA isolation according to the Introvigen protocol (Invitrogen, Carlsbad, CA) and 
measured using a Bio-Rad DC protein assay (Bio-Rad, Richmond, CA, USA). Aliquots of protein (100 $\mu \mathrm{g}$ of protein each) were resolved on a 10\% SDS-PAGE gel and transferred to a polyvinylidene difluoride membrane (Millipore, Medford, MA, USA). The membrane was incubated with a primary antibody overnight at $4{ }^{\circ} \mathrm{C}$ and then with a secondary antibody conjugated with alkaline phosphatase ( $1 \mathrm{~h}$ at room temperature), which was detected by a chemiluminescence method. The following polyclonal primary antibodies were used: anti-human TLR7 (1:300, IMGENEX, San Diego, CA), anti-human CTSS (1:400, Abcam Inc), anti-human beta-action (1:10000, Abcam Inc).

\section{Statistics}

The statistical significance of real-time results was examined with the nonparametric Mann-Whitney test, using GraphPad Prism 4. In the experiment, $p$ values $<0.05$ were considered significantly different between the lesions group and the normal artery group.

\section{Availability \& requirements}

SAM software package: http://www-stat.stanford.edu/ $\sim$ tibs/SAM/

Cluster3.0 and Treeview1.1.0 software: http://bon sai.ims.u-tokyo.ac.jp/ mdehoon/software/cluster/ sofe.htm

MAPPFinder (Gene MicroArray Pathway Profiler): http:// www.genmapp.org

\section{DAVID: http://david.abcc.ncifcrf.gov/}

Xie et al Supplementary Information: http:// www.broad.mit.edu/seq/HumanMotifs/

NCBI web site: ftp://ftp.ncbi.nlm.nih.gov/gene/DATA/ gene2refseq.gz

\section{Authors' contributions}

SJF, experiment design and conduction, and manuscript drafting; HGZ, clinical sample processing; JTS, transcription factor enrichment analysis; AA, WPK, KC and LO-M, data analysis and manuscript revising; JQZ, data analysis; YZD, data analysis and literature support; JZ and MEJ, experimental design and manuscript revising; JGJ, experiment design, data analysis and manuscript revising. All the authors read and approved this version of the manuscript.

\section{Additional material}

\section{Additional File 1}

Table 1. 366 differentially expressed genes in intermediate lesions relative to normal femoral arteries. Affymetrix Probe Set ID, Gene Title, Gene Symbol, GO Biological Process, GO Molecular Function, GO Cellular Component, Unigene, Entrez Gene, Ensembl, Chromosome Number, Socre (d), Fold Change and q-value(\%) which is the lowest FDR are listed in the table.

Click here for file

[http://www.biomedcentral.com/content/supplementary/14712164-9-369-S1.xls]

\section{Additional File 2}

Table 2. Immune-related genes in intermediate lesions relative to normal femoral arteries, Affymetrix Probe Set ID, Gene Title, Gene Symbol, GO Biological Process, GO Molecular Function, GO Cellular Component, Unigene, Entrez Gene, Ensembl, Chromosome Number, Socre(d), Fold Change and q-value(\%) which is the lowest FDR are listed in the table. Click here for file

[http://www.biomedcentral.com/content/supplementary/14712164-9-369-S2.xls]

\section{Additional File 3}

Table 3. Cell component and molecular function categories overrepresented in intermediate lesions, the first half of the table indicates categories highly significant for up-regulated genes; the second half of the table shows categories highly significant for down-regulated genes. The calculated $\mathrm{p}$-values and Z-scores for each category are shown.

Click here for file

[http://www.biomedcentral.com/content/supplementary/1471-

2164-9-369-S3.doc]

\section{Additional File 4}

Table 4. 447 differentially expressed genes in advanced lesions relative to normal femoral arteries. Affymetrix Probe Set ID, Gene Title, Gene Symbol, GO Biological Process, GO Molecular Function, GO Cellular Component, Unigene, Entrez Gene, Ensembl, Chromosome Number, Socre (d), Fold Change and q-value(\%) which is the lowest FDR are listed in the table.

Click here for file

[http://www.biomedcentral.com/content/supplementary/1471-

2164-9-369-S4.xls]

\section{Additional File 5}

Table 5. Immune-related genes in advanced lesions relative to normal femoral arteries, Affymetrix Probe Set ID, Gene Title, Gene Symbol, GO Biological Process, GO Molecular Function, GO Cellular Component, Unigene, Entrez Gene, Ensembl, Chromosome Number, Socre(d), Fold Change and q-value(\%) which is the lowest FDR are listed in the table. Click here for file

[http://www.biomedcentral.com/content/supplementary/14712164-9-369-S5.xls] 


\section{Additional File 6}

Table 6. Cell component and molecular function categories overrepresented in advanced lesions, the first half of the table indicates categories highly significant for up-regulated genes; the second half of the table shows categories highly significant for down-regulated genes. The calculated $\mathrm{p}-$ values and Z-scores for each category are shown.

Click here for file

[http://www.biomedcentral.com/content/supplementary/14712164-9-369-S6.doc]

\section{Additional File 7}

Table 7. 68 commonly up-regulated genes in intermediate lesions and advanced lesions, Affymetrix Probe Set ID, Gene Title, Gene Symbol, GO Biological Process, GO Molecular Function, GO Cellular Component, Unigene, Entrez Gene, Ensembl, Chromosome Number, Socre(d), Fold Change and q-value(\%) which is the lowest FDR are listed in the table. Click here for file

[http://www.biomedcentral.com/content/supplementary/1471 2164-9-369-S7.xls]

\section{Additional File 8}

Table 8. 614 differentially expressed genes in disease progression. Affymetrix Probe Set ID, Cluster ID, Gene Title, Gene Symbol, GO Biological Process, GO Molecular Function, GO Cellular Component, Unigene, Entrez Gene, Ensembl, Chromosome Number, Socre(d), Contrast which is the standardized mean difference between the gene's expression in that class versus its overall mean expression, and q-value(\%) which is the lowest FDR are listed. In the table, contrast1, 2 and 3 represent the standardized mean difference in normal femoral arteries, intermediate lesions, and advanced lesions, respectively.

Click here for file

[http://www.biomedcentral.com/content/supplementary/14712164-9-369-S8.xls]

\section{Additional File 9}

Table 9. The detail GO overrepresented categories for disease progression in each cluster, the calculated $\mathrm{p}$-values and Z-scores for each category are shown in the table.

Click here for file

[http://www.biomedcentral.com/content/supplementary/14712164-9-369-S9.doc]

\section{Additional File 10}

Table 10. 72 putative AP-1 target genes in disease progression. Affymetrix Probe Set ID, Gene Title, Gene Symbol, GO Biological Process, GO Molecular Function, GO Cellular Component, Unigene, Entrez Gene, Ensembl, Chromosome Number, Socre(d), Contrast, and q-value(\%) are listed.

Click here for file

[http://www.biomedcentral.com/content/supplementary/1471 2164-9-369-S10.xls]

\section{Additional File 11}

Table 11. 55 putative CREB target genes in disease progression. Affymetrix Probe Set ID, Gene Title, Gene Symbol, GO Biological Process, GO Molecular Function, GO Cellular Component, Unigene, Entrez Gene, Ensembl, Chromosome Number, Socre(d), Contrast, and q-value(\%) were listed.

Click here for file

[http://www.biomedcentral.com/content/supplementary/14712164-9-369-S11.xls]

\section{Additional File 12}

Table 12. The primer sequences of selected genes for real-time PCR. Click here for file

[http://www.biomedcentral.com/content/supplementary/14712164-9-369-S12.doc]

\section{Acknowledgements}

This work was supported in part by grants from Science and Technology Commission of Shanghai Municipality (project 044319209, 04JCI 4084 and 06QA 14059), Chinese National Key Program for Basic Research (973: 2006CB910405), Chinese National High Tech Program (863: 2007AA02Z335 and 863: 2006AA02Z332), 100-Talent and Knowledge Innovation Programs of Chinese Academy of Science (J. Z), and the Komen Foundation (FAS0703850, WPK and LOM).

\section{References}

I. Lumsden AB, Rice TW, Chen C, Zhou W, Lin PH, Bray P, Morrisett I, Nambi V, Ballantyne C, Peripheral Arterial Occlusive Disease: Magnetic Resonance Imaging and the Role of Aggressive Medical Management. World J Surg 2007, 3 I:695-704.

2. Liles Debra R, Kallen Michael A, Petersen Laura A, Bush Ruth L: Quality of Life and Peripheral Arterial Disease. Journal of Surgical Research 2006, I36:294-301

3. Zhang SH, Reddick RL, Piedrahita JA, Maeda N: Spontaneous hypercholesterolemia and arterial lesions in mice lacking apolipoprotein E. Science 1992, 258:468-47I.

4. Nakashima Y, Plump AS, Raines EW, Breslow JL, Ross R: ApoE-deficient mice develop lesions of all phases of atherosclerosis throughout the arterial tree. Arterioscler Thromb 1994, | 4: I33-140.

5. Heller EA, Liu E, Tager AM, Yuan Q, Lin AY, Ahluwalia N, Jones K, Koehn SL, Lok VM, Aikawa E, Moore KJ, Luster AD, Gerszten RE: Chemokine CXCLIO promotes atherogenesis by modulating the local balance of effector and regulatory $\mathbf{T}$ cells. Circulation 2006, I |3:2301-2312.

6. Combadiere C, Potteaux S, Gao JL, Esposito B, Casanova S, Lee EJ, Debre P, Tedgui A, Murphy PM, Mallat Z: Decreased atherosclerotic lesion formation in CX3CRI/apolipoprotein E double knockout mice. Circulation 2003, 107:1009-1016.

7. Galkina Elena, Harry Brian L, Ludwig Andreas, Liehn Elisa A, Sanders John M, Bruce Anthony, Weber Christian, Ley Klaus: CXCR6 Promotes Atherosclerosis by Supporting T-Cell Homing, Interferon- $\gamma$ Production, and Macrophage Accumulation in the Aortic Wall. Circulation 2007, I 16:1801-I8I I.

8. Aslanian Ara M, Charo Israel F: Targeted Disruption of the Scavenger Receptor and Chemokine CXCLI6 Accelerates Atherosclerosis. Circulation 2006, I I 4:583-590.

9. Hansson Göran K, Robertson Anna-Karin L, Söderberg-Nauclér Cecilia: INFLAMMATION AND ATHEROSCLEROSIS. Annual Review of Pathology: Mechanisms of Disease 2006, I :297-329.

10. Ashley EA, Ferrara R, King JY, Vailaya A, Kuchinsky A, He X, Byers B, Gerckens U, Oblin S, Tsalenko A, Soito A, Spin JM, Tabibiazar R, Connolly AJ, Simpson JB, Grube E, Quertermous T: Network analysis of human in-stent restenosis. Circulation 2006, I | 4:2644-2654.

II. Singhal S, Wiewrodt R, Malden LD, Amin KM, Matzie K, Friedberg J, Kucharczuk JC, Litzky LA, Johnson SW, Kaiser LR, Albelda SM: Gene expression profiling of malignant mesothelioma. Clin Cancer Res 2003, 9:3080-3097.

12. Lenk GM, Tromp G, Weinsheimer S, Gatalica Z, Berguer R, Kuivaniemi $\mathrm{H}$ : Whole genome expression profiling reveals a significant role for immune function in human abdominal aortic aneurysms. BMC Genomics 2007, 8:237-249.

13. Papaspyridonos Marianna, Smith Alberto, Burnand Kevin G, Taylor Peter, Padayachee Soundrie, Suckling Keith E, James Christian H, Greaves David R, Patel Lisa: Novel Candidate Genes in Unstable Areas of Human Atherosclerotic Plaques. Arterioscler Thromb Vasc Biol 2006, 26: 1837-1844. 
14. Potteaux S, Combadiere C, Esposito B, Casanova S, Merval R, Ardouin P, Gao JL, Murphy PM, Tedgui A, Mallat Z: Chemokine receptor CCRI disruption in bone marrow cells enhances atherosclerotic lesion development and inflammation in mice. Mol Med 2005, I I: I6-20.

I5. Lötzer K, Funk CD, Habenicht AJ: The 5-lipoxygenase pathway in arterial wall biology and atherosclerosis. Biochim Biophys Acto 2005, I 736:30-37.

16. Ström A, Franzén A, Wängnerud C, Knutsson AK, Heinegård D, Hultgårdh-Nilsson A: Altered vascular remodeling in osteopontindeficient atherosclerotic mice. J Vasc Res 2004, 41:314-322.

17. Yan SF, Harja E, Andrassy M, Fujita T, Schmidt AM: Protein kinase $C$ beta/early growth response-I pathway: a key player in ischemia, atherosclerosis, and restenosis. I Am Coll Cardiol 2006, 48:A47-55.

18. Bonta PI, van Tiel CM, Vos M, Pols TW, van Thienen JV, Ferreira V, Arkenbout EK, Seppen J, Spek CA, Poll T van der, Pannekoek H, de Vries C]: Nuclear receptors Nur77, NurrI, and NOR-I expressed in atherosclerotic lesion macrophages reduce lipid loading and inflammatory responses. Arterioscler Thromb Vasc Biol 2006, 26:2288-2294

19. Manea Adrian, Manea Simona A, Gafencu Anca V, Raicu Monica, Simionescu Maya: AP-ITranscriptional Regulation of NADPH Oxidase in Human Aortic Smooth Muscle Cells Role of p22phox Subunit. Arterioscler Thromb Vasc Biol 2008, 28:878-885

20. Kastl SP, SpeidI WS, Kaun C, Katsaros KM, Rega G, Afonyushkin T, Bochkov VN, Valent P, Assadian A, Hagmueller GW, Hoeth M, de Martin R, Ma Y, Maurer G, Huber K, Wojta J: In human macrophages the complement component $\mathrm{C5}$ a induces the expression of oncostatin M via AP-I activation. Arterioscler Thromb Vasc Biol 2008, 28:498-503.

21. Xiao W, Hodge DR, Wang L, Yang X, Zhang X, Farrar WL: NF-kappaB activates IL-6 expression through cooperation with $\mathrm{C}$ Jun and IL6-AP I site, but is independent of its IL6-NFkappaB regulatory site in autocrine human multiple myeloma cells. Cancer Biol Ther 2004, 3:1007-1017.

22. Lukiw WJ, Pelaez RP, Martinez J, Bazan NG: Budesonide epimer R or dexamethasone selectively inhibit platelet-activating factor-induced or interleukin I beta-induced DNA binding activity of cis-acting transcription factors and cyclooxygenase-2 gene expression in human epidermal keratinocytes. Proc Nat Acad Sci USA 1998, 95:39|4-3919.

23. Han JS, Macarak E, Rosenbloom J, Chung KC, Chaqour B: Regulation of Cyr6 I/CCNI gene expression through RhoA GTPase and p38MAPK signaling pathways. Eur J Biochem 2003 270:3408-342I.

24. Healy ZR, Zhu F, Stull JD, Konstantopoulos K: Elucidation of the signaling network of COX-2 induction in sheared chondrocytes: COX-2 is induced via a Rac/MEKKI/MKK7/JNK2/c-JunC/EBPbeta-dependent pathway. Am J Physiol Cell Physiol 2008, 294: CII46-II57.

25. Sands WA, Palmer TM: Regulating gene transcription in response to cyclic AMP elevation. Cell Signal 2008, 20:460-466

26. Gay NJ, Gangloff M, Weber AN: Toll-like receptors as molecular switches. Nat Rev Immunol 2006, 6:693-698.

27. Janeway CA Jr, Medzhitov R: Innate immune recognition. Annu Rev Immunol 2002, 20:197-2I6.

28. Faure E, Thomas L, Xu H, Medvedev A, Equils O, Arditi M: Bacterial lipopolysaccharide and IFN-gamma induce Toll-like receptor $\mathbf{2}$ and Toll-like receptor $\mathbf{4}$ expression in human endothelial cells: role of NF-kappa B activation. J Immunol 200I, 166:2018-2024.

29. Mullick AE, Tobias PS, Curtiss LK: Modulation of atherosclerosis in mice by Toll-like receptor 2. I Clin Invest 2005, I I 5:3 |49-3 I 56.

30. Hasebe Akira, Pennock Nathan D, Mu Hong-Hua, Chan Fok V, Taylor Michael L, Cole Barry C: A microbial TLR2 agonist imparts macrophage-activating ability to apolipoprotein A-I. J Immunol 2006, I 77:4826-4832

31. Machida Noriko, Umikawa Masato, Takei Kimiko, Sakima Nariko, Myagmar Bat-Erdene, Taira Kiyohito, Uezato Hiroshi, Ogawa Yoshihide, Kariya Ken-ichi: Mitogen-activated protein kinase kinase kinase kinase $\mathbf{4}$ as a putative effector of Rap2 to activate the c-Jun N-terminal kinase. J Biol Chem 2004, 279: |57| I-I57|4.

32. Yao Zhengbin, Zhou Guisheng, Wang Xuhong Sunny, Brown Amy, Diener Katrina, Gan Hong, Tan Tse-Hua: A novel human STE20related protein kinase, HGK, that specifically activates the c-
Jun N-terminal kinase signaling pathway. J Biol Chem 1999 274:2I|8-2125.

33. Nelson PR, O'Malley KA, Feezor RJ, Moldawer LL, Seeger JM: Genomic and proteomic determinants of lower extremity revascularization failure: rationale and study design. J Vasc Surg 2007, 45(Suppl A):A82-91.

34. Hernández-Vargas P, Ortiz-Muñoz G, López-Franco O, Suzuki Y, Gallego-Delgado J, Sanjuán G, Lázaro A, López-Parra V, Ortega L, Egido J, Gómez-Guerrero C: Fcgamma receptor deficiency confers protection against atherosclerosis in apolipoprotein $E$ knockout mice. Circ Res 2006, 99: I I88-I 196.

35. Alfonso Christopher, Karlsson Lars: NONCLASSICAL MHC CLASS II MOLECULES. Annu Rev Immunol 2000, I 8: I | 3- | 42.

36. Capey S, Mosedale JG, Berg CW van den: Characterisation of the complement susceptibility of the rat aortic smooth muscle cell line A7r5. Mol Immunol 2007, 44:608-6/4.

37. Walport Mark J: Complement - First of Two Parts. N Engl J Med 200I, 344:1058-1066.

38. Oksjoki R, Laine P, Helske S, Vehmaan-Kreula P, Mayranpaa MI, Gasque P, Kovanen PT, Pentikainen MO: Receptors for the anaphylatoxins $\mathrm{C} 3 \mathrm{a}$ and $\mathrm{C5} \mathrm{a}$ are expressed in human atherosclerotic coronary plaques. Atherosclerosis 2007, 195:90-99.

39. Tabibiazar R, Wagner RA, Ashley EA, King JY, Ferrara R, Spin JM, Sanan DA, Narasimhan B, Tibshirani R, Tsao PS, Efron B, Quertermous T: Signature patterns of gene expression in mouse atherosclerosis and their correlation to human coronary disease. Physiol Genomics 2005, 22:213-226.

40. Seo D, Wang T, Dressman H, Herderick EE, Iversen ES, Dong C, Vata K, Milano CA, Rigat F, Pittman J, Nevins JR, West M, GoldschmidtClermont PJ: Gene expression phenotypes of atherosclerosis. Arterioscler Thromb Vasc Biol 2004, 24: 1922-1927.

4I. Qiu X, Brooks Al, Klebanov L, Yakovlev N: The effects of normalization on the correlation structure of microarray data. $B M C$ Bioinformatics 2005, 6: I20-129.

42. Klebanov Lev, Qiu Xing, Welle Stephen, Yakovlev Andrei: Statistical methods and microarray data. Nature Biotechnology 2007 , 25:25-26.

43. Wu Z, Irizarry RA: Stochastic models inspired by hybridization theory for short oligonucleotide arrays. J Comput Biol 2005 I 2:882-893

44. Xie Xiaohui, Lu Jun, Kulbokas EJ, Golub Todd R, Mootha Vamsi, Lindblad-Toh Kerstin, Lander Eric S, Kellis Manolis: Systematic discovery of regulatory motifs in human promoters and $30 \mathrm{UTRs}$ by comparison of several mammals. Nature 2004, 434:338-345.

45. Rhodes DR, Kalyana-Sundaram S, Mahavisno V, Barrette TR, Ghosh $D$, Chinnaiyan AM: Mining for regulatory programs in the cancer transcriptome. Nat Genet 2005, 37:579-583.

Publish with Biomed Central and every scientist can read your work free of charge

"BioMed Central will be the most significant development for disseminating the results of biomedical research in our lifetime. "

Sir Paul Nurse, Cancer Research UK

Your research papers will be:

- available free of charge to the entire biomedical community

- peer reviewed and published immediately upon acceptance

- cited in PubMed and archived on PubMed Central

- yours - you keep the copyright
BioMedcentral 